\title{
A General Model of Mobile Environments: Simulation Support for Strategic Management Decisions
}

\author{
Volker Gruhn, Thomas Richter* \\ Chair of Applied Telematics / e-Business, Dept. of Computer Science, University of Leipzig \\ Klostergasse 3, 04109 Leipzig, Germany; Phone: +49-341-97-32330, Fax: +49-341-97-32339 \\ \{gruhn, richter\}@ebus.informatik.uni-leipzig.de
}

\begin{abstract}
Since the ability of Workforce Management Systems to handle mobility induced challenges of mobile environments like data-communication cut-offs, reduced network bandwidth, and security concerns improved recently, the optimization efforts of mobile enterprises increasingly focus on the organizational setup of their mobile environment. This includes issues like, e.g., the dimension and staffing of regional subdivisions, qualification balance of the workforce, and resource allocation strategies. While this multitude of possible adjustment parameters for optimization prevents from the analytical prediction of organizational change efforts, simulation is a promising approach to analyze mobile environments and their change.

In this work we present a formal model representing a generalization of mobile environments. This model can be utilized to examine the cost situation and performance of both real mobile enterprises and projected future development scenarios of such enterprises. The model is developed using colored petri nets (CPN) and the software suite CPN Tools. We show that our model is capable of predicting the outcomes of organizational change projects by the utilization of simulation and present a validation of our model based on real-world data of a German gas and power supply.
\end{abstract}

Keywords: Mobile Environments, Enterprise Simulation, Workforce Management, Resource Modeling, Colored Petri Nets

\section{Introduction}

Mobile processes in distributed and/or volatile business environments are increasingly gaining attention

* Corresponding author regarding efforts to improve the efficiency of process execution. Besides the constant pressure any organization experiences to improve its performance by means of change [6], the main technical reason is the increasing availability of high-bandwidth $3 \mathrm{G}$ data communication networks [19]. Process related data can now be processed both downstream (i.e. toward the mobile worker) and upstream (i.e. toward the organization's information systems). This allows for mobile business processes to become controlled centrally.

In this work mobile processes are referred to as business processes of which at least one activity takes place outside the organization's physical bounds - i.e. in the field [23]. Examples of such processes are the maintenance of supplier networks (electrical power, gas, water, etc.), the service of technical equipment at customer's sites, and the monitoring of distributed sites as construction sites [25] or supermarkets (e.g. mystery shopping [39]).

Mobile processes are characterized by a number of attributes differentiating them from non-mobile processes. Among them are the location awareness of activities - i.e. the necessity to have workers travel to the site of the activity's execution - and thus the possibility of data and voice communication cut-offs during process execution. The location awareness of mobile processes represents a challenging restriction for the scheduling of the workforce (the assignment of resources to mobile activities) [22] in comparison to the resource assignment in non-mobile processes [35]. It leads to the necessity to prepare work lists for each worker, to preload data to the workers' mobile devices, and to the consideration of the spatial distribution of skills and competence over time. The latter is forced by the possibility of highly prioritized processes, that require immediate execution by nearby located resources.

From a certain size of the area they cover, such enterprises tend to organize themselves in subdivisions with responsibility for regionally assigned assets and their 
respective business processes. Thus the enterprise administers a number of regional divisions with identical functional processes performed on their respective distributed assets. Such assets can be dedicated predefined customer sites, immobile technical equipment, or a variety of different consumer products at unforeseeable sites - if the company offers appropriate repair services, for instance. Assignments of such assets of responsibility to a subdivision are often based on either historical evolution of the enterprise, administrative structures as counties or states, or natural boundaries like rivers or mountain ranges. The establishment of such assignments may have sensible reasons at first glance but an organization can hardly determine, if the regional assignment of assets, resources, and workers is optimal regarding the organization's cost situation. This situation aggravates in the occasion of mergers or acquisitions, since there is usually no prior knowledge about the performance of the unified workforce. Cost functions may be based on travel distances, duration of technical malfunctions, or the number of workers employed. They strongly vary between organizations based on their respective areas of business.

Due to the fact that the numerous aforementioned constraints of mobile environments, a wide variety of cost functions, and differently targeted workforce scheduling algorithms prevent from analytical prediction of changes to mobile environments, this work aims at the simulation-based investigation of effects of different organizational setups of mobile process environments. Since the necessity to maintain workers' work lists is a core attribute of mobile environments, actually independent business processes may become interdependent by the demand of identical resources. Thus the regional assignment of assets and resources in mobile environments influences and is influenced by all concerned business processes. Therefore approaches to business process simulation for single processes are not fully suitable as a solution of the problem addressed. Moreover the influence of several business processes to modeling and simulation forces the development of a general high-level model of mobile environments, into which models of the different business processes can be integrated as necessary. We introduce such a general model of mobile environments, which can be fed with data of real or designed scenarios and predict the performance and cost-effects of these different scenarios examined. Thus change decisions can be supported by results from the application of the model to the enterprise's current situation and the planned changes. The contribution of our work is such a general model of mobile environments applicable to various domains.

The remainder of this article is organized as follows: section 2 gives an overview of existing enterprise modeling approaches, business process simulation and aspects of mobility. Section 3 introduces the models developed. In section 4 the aforementioned models are utilized to analyze the mobile environment of a German gas and power supply. Section 5 concludes the article and gives directions for further research.

\section{Related Work}

Mobile applications play an important role in efforts to optimize mobile organizations and their business processes. A number of case studies of real-world scenarios examine the effects of such solutions in several industries as in service organizations [16], in the utility industry $[27,34]$, in the construction industry $[8,25]$, in banking services [24], in mobile police work [30], and in the paper industry [18] for example. Much research focuses on dedicated aspects of such applications. In [32] an approach to derive functional and non-functional requirements from the characteristics of the mobile scenario is presented. This work also analyzes the general characteristics of mobile work. Several aspects of mobile tasks' quality concerns are presented in [19] and [23], while [33] outlines and thoroughly analyzes the mobility induced challenges for software engineering as a whole. The authors divide mobility in physical mobility of mobile hosts (workers and / or devices) and logical mobility of code and state. The former is associated with our question for optimal dimensions of administrative regions, while the latter is influenced by scheduling decisions based on the workforce's state. Additionally the authors discuss many different issues of architectural design for mobile applications. [5] propagates the utilization of the Micro-Apache Generic Interface to overcome limitations of web-induced inhibition of decentralization of process definitions. More general approaches to technical and architectural concerns of mobile systems are presented in [12] and [15]. The mobility related research mentioned above focuses either on the independent support of single mobile processes, on the technical and administrative concerns of implementing mobile systems, or on the characteristics of mobility itself.

Regarding business process simulation (BPS) the authors of [31] state, that the simulation of business processes creates an added value in understanding, analyzing, and designing processes by introducing dynamic aspects. They argue for the application of process maps for simulation modeling in Business Process Change (BPC) projects. The application of computer based simulation models of business processes reduces the complexity of organizational analysis and thus con- 
tributes to a higher level of understanding and designing organizational structures [13]. It enables migration from static toward a dynamic process models [1]. Simulation helps to reveal the reasons for unexpected behavior of process execution [7]. Although business process simulation is widely accepted in research communities, an empirical study [26] discovered, that $64 \%$ of the enterprises examined don't use simulation to predict the outcomes of change projects in advance. This may be due to the broad focus of simulation tools which aim to cover most of the problem domains suitable for simulation. In [10] a business process simulation template for the Arena ${ }^{1}$ simulation suite is presented to support appropriate industrial projects. Though BPS is increasingly gaining attention, most approaches focus on single business processes, but only marginally consider the properties of the process execution environment - where several concurrent processes are executed.

Enterprise Modeling (EM) aims to overcome the aforesaid limitations of BPS approaches by considering the organization as a whole with it's complete set of business processes. Regarding the development of methods for the modeling of organizations [3] states that "Since the 80s, two main approaches to Enterprise Integration (EI) have matured: (i) the integration of software applications that support the functionality of business processes; (ii) the definition of Enterprise Models by means of Enterprise Modelling Languages and integration of such models, for understanding, validating and effectively achieving EI". Prevalent enterprise modeling methods are GRAI [11], PERA [38], GERAM [36], and CIMOSA [20]. These approaches mainly focus on either enterprise models for production and manufacturing environments or on the documentation and understanding of organizational structures for the development of Enterprise Resource Planing (ERP) Systems. They are not intended to support mobile environments natively. In [4] an enterprise modeling framework for distributed organizations (CEMF-DO) is proposed. This framework aims at the utilization of the Unified Enterprise Modeling Language (UEML) [37] to gain interoperability of different distributed enterprise models.

Resource allocation for the non-mobile case is thoroughly discussed in [35], where 43 different patterns for the allocation of resources to work items are presented (we use the terms activity and work item in interchangeable manner). The nature of mobile work prevents numerous of the patterns from being utilized in mobile environments, since pull-mechanisms (the work-

1 see http://www.arenasimulation.com ers choose the items to work on) are not suitable due to the demand to decrease the travel times and distances between subsequent work items and the local focus of the single worker. The authors of [29] utilize colored petri nets to model and compare the work distribution mechanisms of three different non-mobile workflow management systems. They distinguish static from dynamic resource allocation and argue for flexible work distribution mechanisms based on their reference model. An approach for the improvement of role-based resource allocation mechanisms by the consideration of unavailability or overloading of workers, context dependent suitability, deadlines, and delegation issues is presented in [21], also focusing on the non-mobile case. A colored petri nets based simulation model for the analysis of non-mobile resource-constrained processes is presented in [28]. This work was the basis and starting point of the simulation and modeling approach for mobile environments we are presenting here. Another initial work for our research is found in [14], where the authors present a simulation based modeling and analysis approach for the performance and cost evaluation of mobile information systems.

\section{Development of the CPN model}

Characteristics of the processes in question Since we want to develop a general model of mobile environments and thus focus on just the mobile part of the organization's processes, we don't need to cover the business process as a whole, but can abstract from the actual business process models. We define a representation of mobile work as illustrated in figure 1 . The marked part of the model contains the relevant process steps for our work.

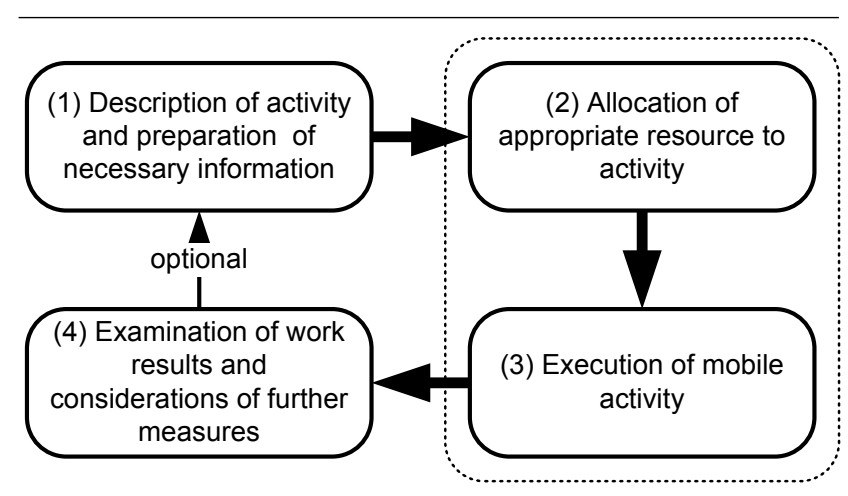

Figure 1. Abstract mobile process

This characterization of mobile processes implies 
several assumptions and attributes to be provided. For the allocation of appropriate resources to activities we need to determine (i) the appropriate qualification (role) of the resource capable to execute the activity and (ii) the allocation (or scheduling) algorithm. To assign a resource to an activity, the resource's role must be in the set of roles that are able to perform the underlying task. Due to the fact, that traveling contributes considerably to the costs of mobile processes, we need to manage work lists which contain a resource's activities for a given period of time (e.g. one day) in chronological order. The relevant attributes of the execution of mobile activities are (i) the geographic location of the asset associated with the activity, (ii) the expected execution time, and (iii) the task to be performed. In addition to approaches modeling non-mobile processes each resource must keep information about its current geographic location and the work items either have to be assigned to a geographic location.

Evaluation objectives Since we focus on the examination of different organizational scenarios of one and the same mobile environment, the model must be able to answer the following questions regarding different setups:

1. What impact has the modification of the dimensions of regional subdivisions (regions) on the overall costs?

2. Is the workforce distribution among the regions suitable to perform all activities in a certain amount of time?

3. Is the workforce's qualification suitable for the kind and amount of activities to perform?

Assuming, that - abstracting from underlying business objectives - travel times and workforce utilization have the most impact on cost changes in mobile environments, we present a short formal description of the according coherences.

Be $W$ the set of all workers and $A$ the set of all assets. Then the sequence $w l_{w}^{d}=\left(a_{1}, \ldots, a_{n}\right)$ with $a_{i} \in A$ is the work list of worker $w \in W$ for day $d$. Let $\operatorname{loc}\left(a_{i}\right)$ define the geographic location of asset $a_{i}$. Then the travel list of worker $w$ for day $d$ is the sequence

$$
t l_{w}^{d}=\left(\operatorname{loc}\left(\operatorname{home}_{w}\right), \operatorname{loc}\left(a_{1}\right), \ldots, \operatorname{loc}\left(a_{n}\right), \operatorname{loc}\left(h_{o m e}\right)\right)
$$

with home ${ }_{w}$ being the home base of worker $w$. Let further $t l(i)$ be the $i$ th element of $t l$ and $\operatorname{travel}\left(l_{1}, l_{2}\right)$ be the travel time between locations $l_{1}$ and $l_{2}$. Then the daily travel time of worker $w$ is calculated as

$$
t_{w, d}^{\text {travel }}=\sum_{i=1}^{n+1} \operatorname{travel}\left(t l_{w}^{d}(i), t l_{w}^{d}(i+1)\right)
$$

Let further $\operatorname{days}(w)$ be the number of working days of worker $w$. Then the overall travel time $t^{\text {travel }}$ can be calculated as

$$
t^{\text {travel }}=\sum_{w=1}^{|W|} \sum_{d=1}^{\operatorname{days}(w)} t_{w, d}^{\text {travel }}
$$

The overall working time $t^{\text {work }}$ can be calculated in a similar way. The total execution time $t^{\text {total }}$ of all activities in the organization is then

$$
t^{\text {total }}=t^{\text {work }}+t^{\text {travel }}
$$

The overall times for a single worker $\left(t_{w}^{\text {total }}, t_{w}^{\text {work }}\right.$, and $\left.t_{w}^{\text {travel }}\right)$ are defined accordingly. Let $R$ be the set of all regions, $r \in R$ be a region, and $W_{r}$ be the set of the workers assigned to region $r$, then the according times for a region are determined by:

$$
\begin{gathered}
t_{r}^{\text {travel }}=\sum_{w=1}^{\left|W_{r}\right|} t_{w}^{\text {travel }} ; \quad t_{r}^{\text {work }}=\sum_{w=1}^{\left|W_{r}\right|} t_{w}^{\text {work }} \\
t_{r}^{\text {total }}=t_{r}^{\text {travel }}+t_{r}^{\text {work }}
\end{gathered}
$$

Let further $A_{r}$ be the set of assets assigned to region $r$, then the region's total and travel efforts per asset are calculated by:

$$
t_{\text {asset }}^{\text {total }}(r)=\frac{t_{r}^{\text {total }}}{\left|A_{r}\right|} ; \quad t_{\text {asset }}^{\text {travel }}(r)=\frac{t_{r}^{\text {travel }}}{\left|A_{r}\right|}
$$

Further comparability of regions can be achieved by the total completion time of all activities in a region (time stamp of the last activity's finish):

$$
t_{r}^{\text {comp }}=\max \left(t_{w}^{\text {total }} \mid w \in W_{r}\right)
$$

and by the average total execution time per worker in each region:

$$
t_{r}^{a v g}=\frac{t_{r}^{\text {total }}}{\left|W_{r}\right|}
$$

Therewith a number of basic evaluation criteria for mobile environments are defined. These are by far not complete, but will be suitable for the validation of our model, which is the aim of this work. In the ongoing evolution of the model (see sec. 5) we will introduce further evaluation criteria.

Overview To develop a general model of mobile environments, we decided to use colored petri nets (CPN) [17] - an extension of petri nets - since they have a complete formal basis, and tools for the modeling and simulation are available. As modeling tool we used CPN Tools $^{2}$.

2 see http://wiki.daimi.au.dk/cpntools/cpntools.wiki 
The CPN model consists of several hierarchically associated parts, which represent different aspects of the mobile resource-constrained process environment. The model consists of a main part (TopLevel) containing subparts of which the most important are the Scheduler and the mobile workers' state model (ResourceState).

The model discussed here is independent from the structure of the business processes, since only mobile tasks are analyzed. Figure 2 shows the top level of the CPN model with its parts LoadData, Generator, ResourceState and Scheduler. LoadData performs the setup of an organisation's specific environment and initializes the simulator by loading the data of the process environment (Assets, resources (Workers), and Regions) from text files into certain places of the net. This data will then be used by the other parts of the model. The Generator generates work items, which will be assigned to mobile resources by the scheduler. The Scheduler assigns work items so that each resource has a daily work list. After having a complete schedule (i.e. at least 28.800 seconds or 8 hours of work time and travel time scheduled) a resource begins to flow through the ResourceState part of the model conducting the assigned activities.

Module Scheduler In mobile processes the assignment of resources and work items is restricted by both the skill-match of resources and work items and by their respective geographic location. Besides the resource's utilization the minimization of travel efforts is a main target of workforce scheduling. Therefore it is necessary to create a flexible model which allows to easily switch the scheduling algorithm for comparison.

The module Scheduler (fig. 3) works as follows. First the arriving workitems (NewWorkitem) are assigned to the appropriate roles able to fulfill the work item's underlying task (stored in place TaskRoles). Transition inputRequest adds the created request to a list which presents all pending requests of exactly one region (place Pending). A region represents a part of the area covered by the data. This supports either the altering of the regional structure of real world organizations in different simulation runs and parallel examination of several regions in one and the simulation run. Accordingly each work item and each resource have an attribute region, which is matched by the scheduler in the transition schedule. Since ad-hoc assignment of resources is not appropriate in mobile processes, the scheduler has to predict durations for work and travel, which are added to the resource's corresponding attributes in transition addServiceTime by retrieving the standard working times from place WorkingTimes. The request containing the work item is appended to the resource's list of work items (its schedule).

We have chosen the nearest neighbor algorithm [9] in the first place, which is by far not the best suited algorithm, but easy to implement in CPN ML and gives acceptable and reasonable results. Our first aim was to find out, if the CPN approach is feasible to cover the problem domain at all. The scheduling algorithm is intended to find near to optimal assignments of resources and work items. An optimal assignment is understood as an assignment where all work items are completed and travel times are minimized. Further work (see section 5) aims at the implementation of additional allocation algorithms. The module contains three helper places: RegionCount, Wait, and UpdatedWorker.

Functions of Scheduler The nearest neighbor scheduling algorithm was implemented in the CPN ML (a Standard ML sibling) functions findFirstMatchingRequest, unboundResourceMatches, findClosestRequest, and assignClosestRequest whose names speak for their respective functionality. Helper functions (updateWorker, updateTimePlanned) were implemented to improve the visual simplicity of the model.

Module ResourceState In this module the actual work and traveling is performed. Therefore resources with their respective work lists flow through the model depicted in figure 4.

Since the place Ready is identical to the place Worker of the modules TopLevel and Scheduler, either partly and fully scheduled workers show up in the module ResourceState. To prevent workers from starting their daily work with an incomplete schedule (read: a schedule, which covers less than eight hours), a guard protects the transition startTravel. This guard uses information about the pending requests (place Pending) and the resource's already scheduled working time. As soon as a schedule is completed by the scheduler (expected duration of $8 \mathrm{hrs}$ or no requests left in the corresponding region), the resource enters the place Traveling whilst the according travel time is added to the resource's individual time stamp. Each resource stores its geographic location in the attribute geo. Locations are represented by longitude and latitude values. Times for traveling from the current position to the position of the first work item in the schedule are calculated by assuming, that the resource travels on the direct connection (on a sphere) of the two locations at a speed of $30 \mathrm{~km} / \mathrm{h}$. This assumption proved to be sensible in comparison with a reduced set of $100(1,3 \%)$ representative locations where the travel times were calculated by real route planning systems (Google Maps ${ }^{3}$

3 see http://maps.google.com 


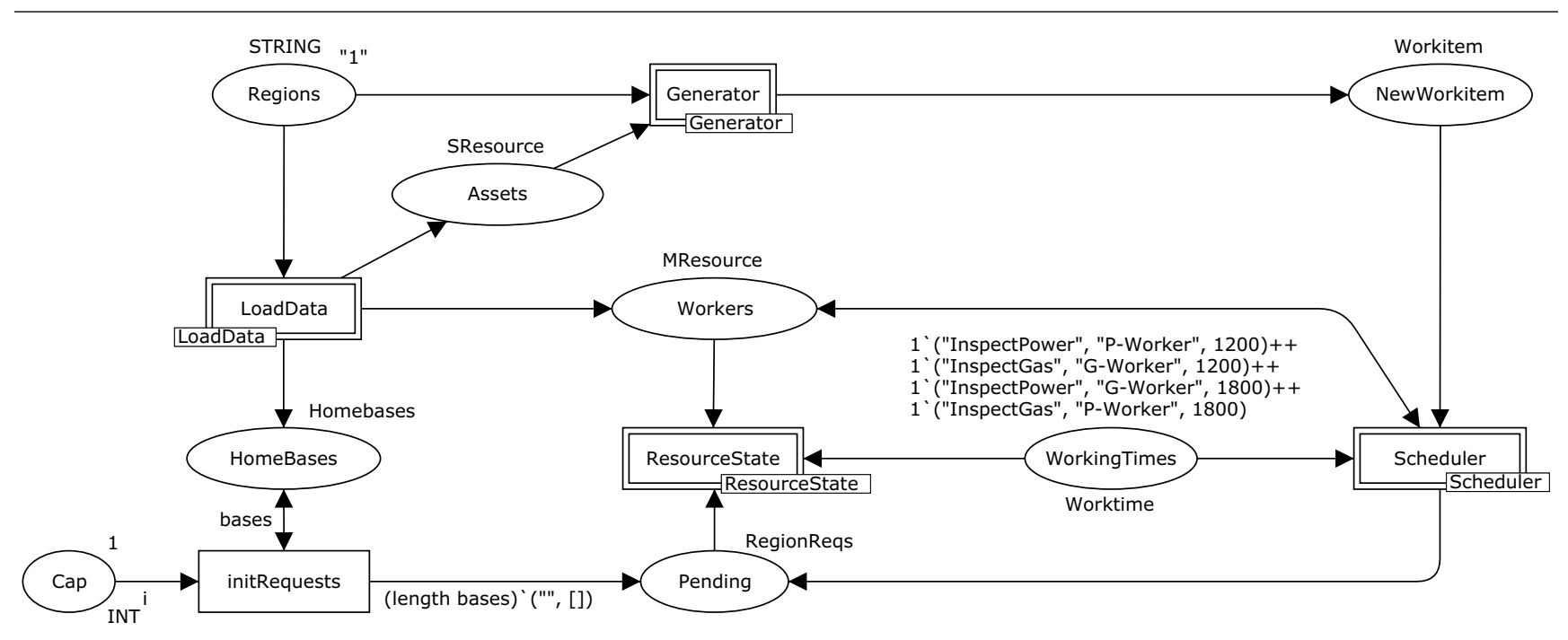

Figure 2. Top Level

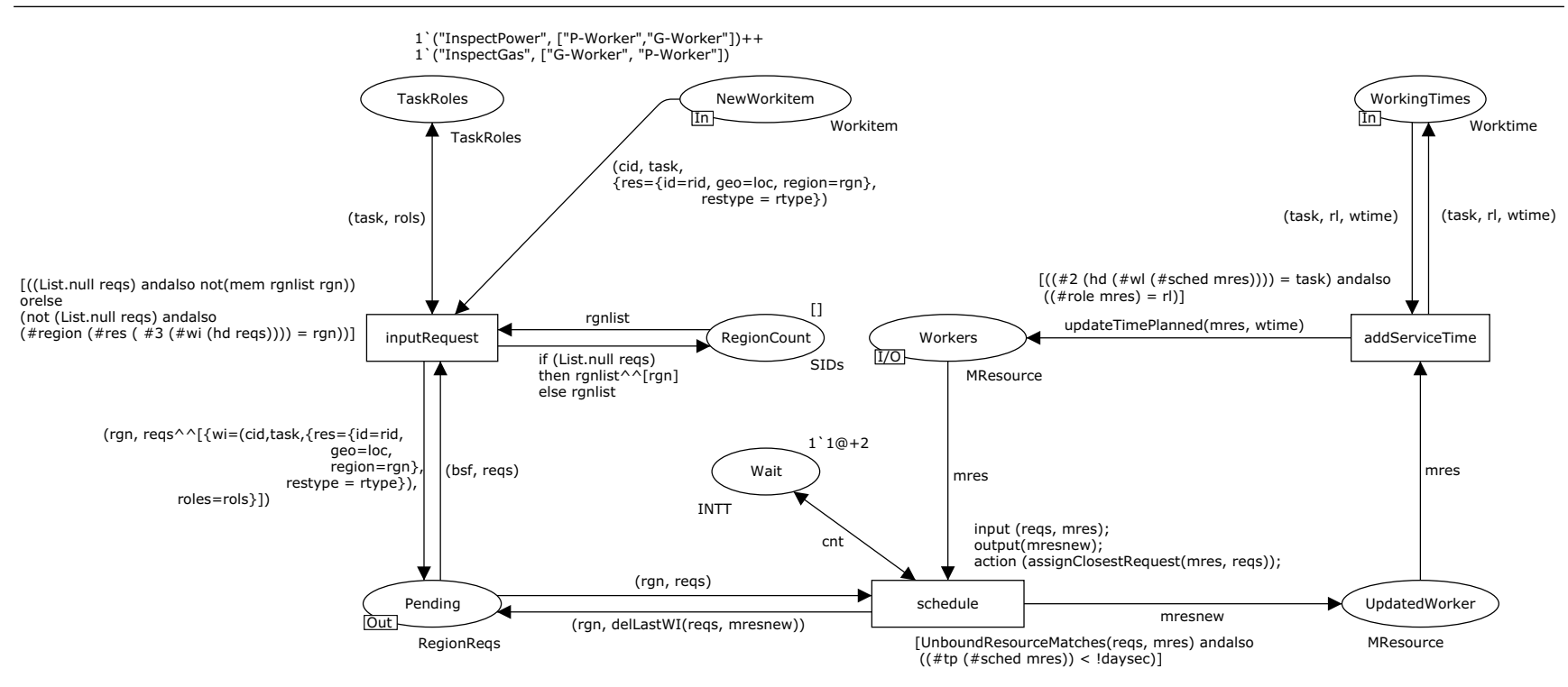

Figure 3. Scheduler

and ViaMichelin ${ }^{4}$ ). The locations for the test were randomly selected from the real simulation data. For different organizational setups the average speed may have to be adjusted, which the model is already prepared for. Nonetheless we are currently considering the integration of a real route planning system for the purpose of the simulations. The application of a symbolic location model [2] to the problem is inappropriate since the

4 see http://www.viamichelin.com locations of activities are not a determined set but unforeseeable.

After reaching the site of the activity to be performed, the working time is added to the resource's time stamp in the transition startJob. The working time is assumed to be equally distributed in an interval of 0.8 to 1.2 times the standard working time for the activity's underlying task and the worker's resource type. The working times differ between different roles for the same task. Since we want to examine different 


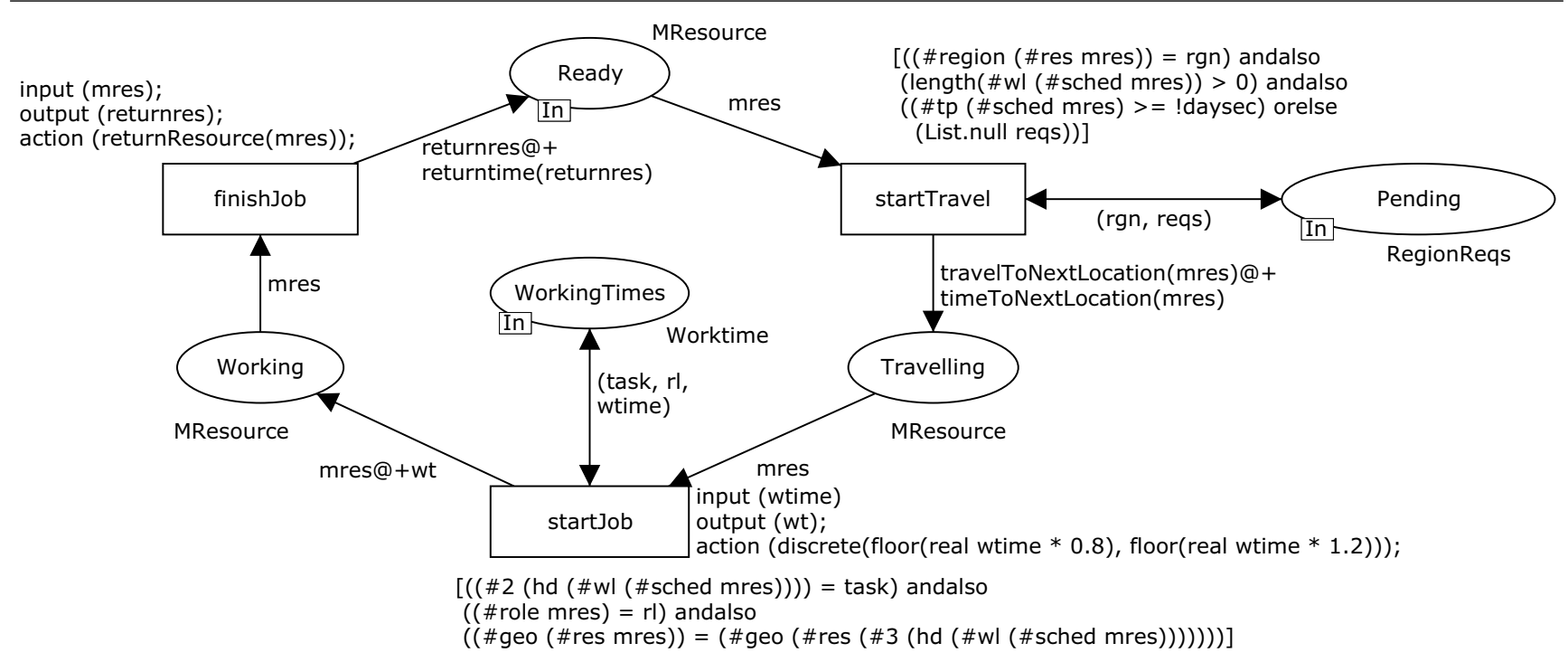

Figure 4. Resource State

administrative setups of an organization we can model worker's different levels of experience in different domains (place WorkingTimes). We assume, that for instance a worker who is genuinely skilled in maintaining power equipment and who was upgraded to maintain gas equipment will need longer for tasks in the gas domain than a genuine worker of that domain for the same tasks.

After finishing its work (leaving place Working) a worker can either travel to the next working site or return to its home base, which could either be the organization's headquarter or the worker's place of accommodation. The worker is set to return home not before its work list is empty, which means, that the daily schedule is completely performed. The transition finishJob calculates and adds the appropriate travel time to the worker's time stamp and adjusts the worker's flextime amount. The calculation of the flextime is necessary to perform the traveling to the working site in the morning and back home in the evening often enough. So overtimes are subtracted from the next day's time budget of the worker. Since the Scheduler can access the place Ready/Workers it can assign requests to the worker for the next day.

Functions of ResourceState The functions implemented for ResourceState are mainly helper functions for the calculation of times with respect to travel distances. As already stated, the time necessary to relocate between working sites or home bases is currently based on a speed assumption for the direct spherical arc between two locations. Locations are stored as tuples of longitude and latitude of their positions on Earth. A sphere is a valid simplification of Earth's surface for small distances $(<100 \mathrm{~km})$. This functionality is implemented in distGeo, calcTravelTime, timeToNextLocation, and travelToNextLocation. The return to the resource's base takes place as soon as the daily work list is empty. Since we assume, that the travel efforts in the morning and the evening have significant influence on the total cost of mobile processes, it is necessary to model working days. This functionality is implemented in returnResource and calcReturnTime. The model we use for the representation of working times is threefold. Each resource has assigned attributes for the overtime worked (ot), for the time stamp of its working day's start (ds), and for the approximately scheduled time (tp). tp is used by the scheduler to keep track on when a resource's daily time budget is fully covered by its work list. Since overtimes are deducted from the daily budget, each resource has an individual daily budget, except for the first day of service. The cumulated overtime is calculated by

$$
o t=n o w+t_{\text {return }}-d s-\text { budget }_{\text {std }}+\text { ot }_{\text {old }}
$$

with now being the current timestamp, $t_{\text {return }}$ being the time to travel back home, budget std $_{\text {being a }}$ worker's daily standard budget ( $8 \mathrm{hrs}$ ), and ot old being the worker's already achieved overtime. The next day's budget is then determined as follows:

$$
\text { budget }_{n e w}=\text { budget }_{\text {std }}-\text { ot }
$$

Color Sets The color sets are defined as follows: 


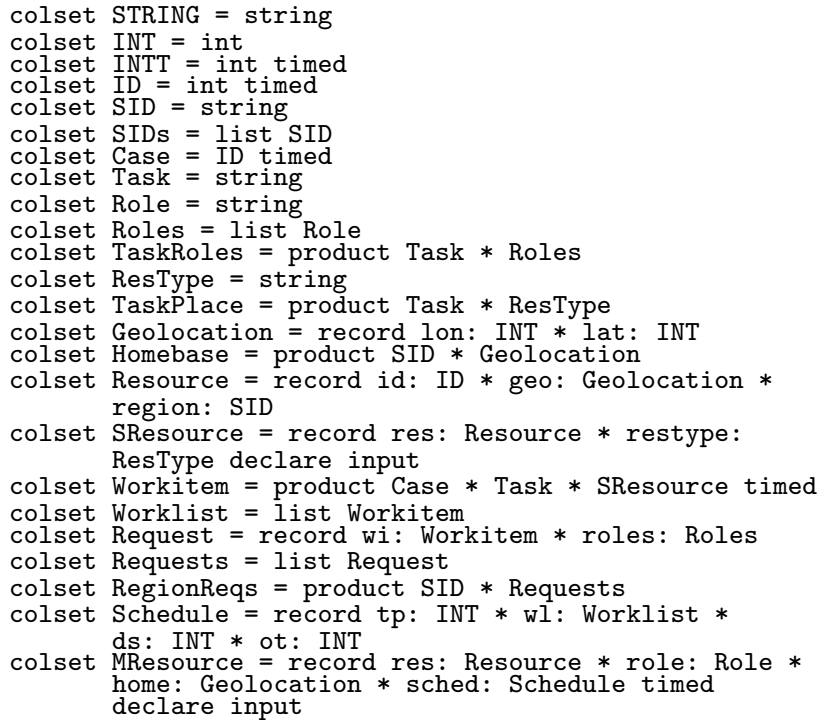

\section{Application of the CPN model}

To validate, if the model described above is capable of representing real-world mobile environments, we applied it to the data of a German power and gas supply. The company covers a rural area of $7.000 \mathrm{~km}^{2}$ and serves about 500,000 customers. The company is the result of a recent merger of the local power and gas suppliers, roughly covering the same area. The power domain has 19 regional subdivisions and 115 workers, while the gas domain has 9 regional subdivisions and 85 workers. For historical reasons, each region of the power domain can be mapped topographically to exactly one region of the gas domain (fig. 5).

Given, that the number of employees in network maintenance will either be constant or decrease over the next years, we examined five different organizational scenarios, which were considered by the company's management. The aim was to find out, if the workers are well distributed over the regions and if it makes sense, to educate power workers for gas domain jobs and vice versa. We identified the following cases to examine:

(1) Current situation: The domains will be strictly separated, meaning, that workers of either domain will only perform work items of their respective domain.

(2) Dimensions of regions: Regions of the power domain are merged such that they cover identical areas as the regions of the gas domain. The responsibilities remain separated as in case 1.

(3) Generalists vs. specialists (partly): If one domain is significantly more stressed, workers of one domain work in the other domain to help decrease work-

\begin{tabular}{ll|ll|ll}
\hline G1: & P1, P2 & G4: & P8 & G7: & P12, P13, P19 \\
G2: & P3, P4 & G5: & P9, P10 & G8: & P14, P15 \\
G3: & P5, P6, P7 & G6: & P6 & G9: & P16, P17, P18
\end{tabular}

Table 1. Mappings of power (P) to gas (G) regions

load. This case is optional.

(4) Generalists vs. specialists: Workers work in either domain. This helps to figure out if the increased working time is compensated by probably less travel time.

(5) Resource distribution: If the worker-to-asset ratio and the overall completion time are both higher than that of other regions, workers are transferred from other regions an thus have a completely new home location.

For the assessment of the simulation approach standardized, annually repeated processes were selected. In the power domain 5,816 power substations and in the gas domain 1,933 slide valves and regulators have to be inspected from January until March. These processes are well suited for the examination of the model, because inspection is an easy to understand and easy to learn task.

Table 2 shows the simulation results. The average total working time is denoted as $t_{a v g}^{w o r k}$, the average total travel time is denoted as $t_{\text {avg }}^{\text {travel }}$, and the average total execution time of the activities performed is denoted as $t_{a v g}^{\text {total }}$. Furthermore the percentage of overall travel, the total execution time per asset $\left(t_{\text {asset }}^{\text {total }}\right)$, and the travel time per asset $\left(t_{\text {asset }}^{\text {travel }}\right)$ are given. It can be stated (see table 1 and fig. 5), that for the cases 1 and $2 t_{\text {asset }}^{\text {travel }}$ of the gas domain is significantly higher than of the power domain. Case 2 shows, that just the unification of the power regions to match the gas regions has no significant impact on the travel effort. As a result of the cases 1 and 2 we have chosen for case 3 to let the workers of the power domain perform activities in the gas domain, aiming at the reduction of travel effort. We assumed, that it takes these workers 20 minutes to perform inspections of assets of their own domain and 30 minutes for assets of the gas domain (place WorkingTimes in fig. 2). This strategy shows almost no impact on the overall performance. Case 4 shows the results for workers performing activities in each domain. As expected, the travel effort drops dramatically, while the total execution time increases by almost $15 \%$ when we assume, that workers need 30 minutes for tasks in the other domain $\left(4_{1800}\right)$. When assuming execution times of 25 minutes $\left(4_{1500}\right)$ and 22.5 minutes $\left(4_{1350}\right)$ 

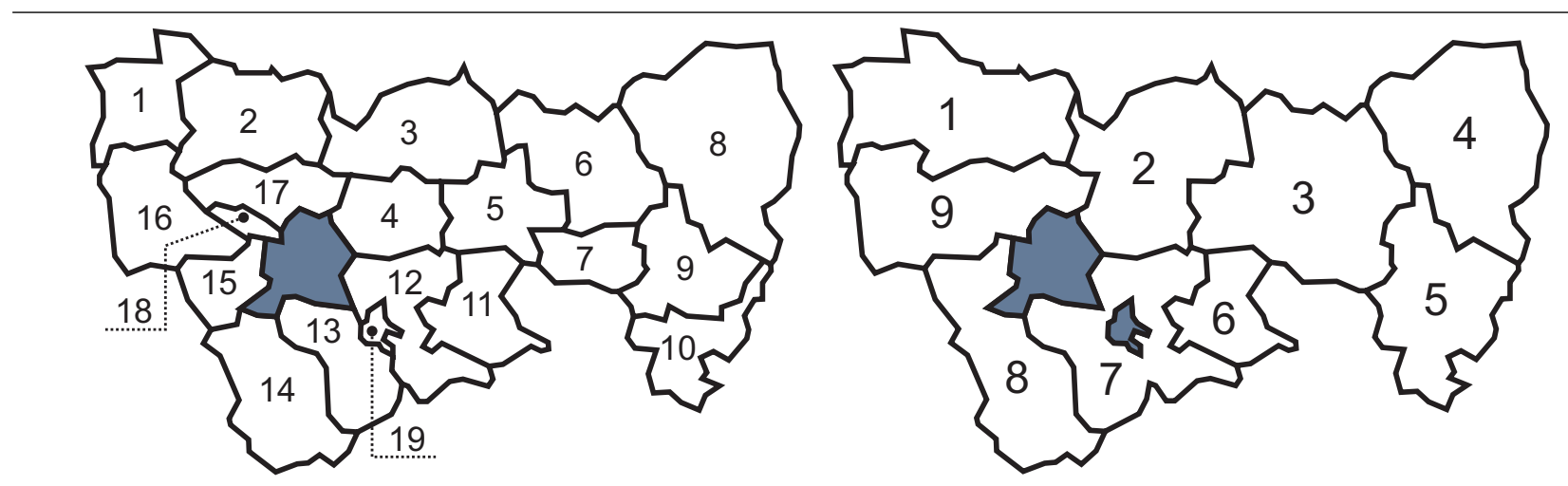

Figure 5. Regions: power (left) and gas (right)

\begin{tabular}{|c|c|c|c|c|c|c|c|c|c|}
\hline Case & Domain & Workers & Assets & $\begin{array}{c}t_{a v g}^{w o r k} \\
(\mathrm{hrs})\end{array}$ & $\begin{array}{c}t_{\text {avg }}^{\text {travel }} \\
(\mathrm{hrs})\end{array}$ & $\begin{array}{c}t_{\text {avg }}^{\text {total }} \\
(\mathrm{hrs})\end{array}$ & $\begin{array}{r}\text { travel } \\
(\%)\end{array}$ & $\begin{array}{r}t_{\text {asset }}^{\text {total }} \\
\text { (mins) }\end{array}$ & $\begin{array}{l}t_{\text {asset }}^{\text {travel }} \\
\text { (mins) }\end{array}$ \\
\hline \multirow{3}{*}{1} & Power & 115 & 5816 & 1937.5 & 224.7 & 2162.2 & 10.4 & 22.31 & 2.32 \\
\hline & Gas & 85 & 1933 & 644.1 & 118.8 & 762.9 & 15.6 & 23.68 & 3.69 \\
\hline & total & 220 & 7749 & 2581.6 & 343.5 & 2925.1 & 11.7 & 22.65 & 2.66 \\
\hline \multirow{3}{*}{2} & Power & 115 & 5816 & 1936.5 & 220.8 & 2157.3 & 10.2 & 22.26 & 2.28 \\
\hline & Gas & 85 & 1933 & 644.1 & 118.8 & 762.9 & 15.6 & 23.68 & 3.69 \\
\hline & total & 220 & 7749 & 2580.6 & 339.6 & 2920.2 & 11.6 & 22.61 & 2.63 \\
\hline 3 & total & 220 & 7749 & 2619.8 & 345.4 & 2965.2 & 11.7 & 22.96 & 2.67 \\
\hline $4_{1800}$ & total & 220 & 7749 & 3128.4 & 277.3 & 3405.7 & 8.1 & 26.37 & 2.15 \\
\hline $4_{1500}$ & total & 220 & 7749 & 2858.6 & 272.3 & 3131.0 & 8.7 & 24.24 & 2.11 \\
\hline $4_{1350}$ & total & 220 & 7749 & 2721.3 & 269.7 & 2991.2 & 9.0 & 23.16 & 2.09 \\
\hline 5 & Power & 115 & 5816 & 1937.2 & 222.9 & 2160.1 & 10.3 & 22.28 & 2.30 \\
\hline
\end{tabular}

instead, we could figure out, that the training of generalists will have advantages only if their working time is less than $12.5 \%$ higher than that of specialists of the appropriate domain. Case 5 shows the same results as case 1 regarding travel effort.

When examining the simulation results of case 1 , we found, that the total completion times (when the last activity is finished) of the 19 regions vary strongly ( $t_{\text {case } 1}^{\text {comp }}$ in fig. 6 and table 3 ). Thus for case 5 we calculated a new distribution of the 115 workers to the 19 regions, such that the asset-to-worker-ratio is almost equal for different regions. Figure 6 shows, that as a result the total completion times $t_{\text {case }}^{\text {comp }}$ are almost equal, while the average completion times $t_{\text {case } 5}^{\text {avg }}$ per worker oscillate less than in case $1\left(t_{\text {case } 1}^{a v g}\right)$.

The outcomes of the simulations seem to indicate that the spatial distribution of mobile activities has only small influence on the performance of the mobile workforce. This is not surprising, since we have chosen processes, that don't have unforeseeable arrival rates, and the tasks are performed such that each asset of the organization is visited exactly once. Thus the to- tal completion times of case 5 must be almost equal between different regions, since they state, that the last activity is finished after about 24 hours (or 3 days). Naturally most of the workers are finishing their tasks before the last activity. Their efforts are shown in the average completion times, which are different between regions. Nonetheless these expectable results validate the model to be suitable for the simulation of mobile environments.

As an outcome of the simulations, a mix of the scenarios 2 and 5 is considered to be realized in future change projects. The examination of scenario 4 validated, that the already made decision to postpone the training of generalists was reasonable.

\section{Conclusions and Future Work}

When mobile organizations improve their business by means of business process reengineering, not just the processes themselves but the environment influences design decisions. Even independently from business process change, process environments are subject 


\begin{tabular}{|c|c|c|c|c|c|c|c|c|c|}
\hline \multirow[t]{2}{*}{ Region } & \multirow[t]{2}{*}{$\operatorname{Assets}(|A|)$} & \multicolumn{4}{|c|}{ Case 1} & \multicolumn{4}{|c|}{ Case 5} \\
\hline & & Workers $\left(\left|W^{1}\right|\right)$ & $\frac{|A|}{\left|W^{1}\right|}$ & $\begin{array}{c}t_{r}^{a v g} \\
(\mathrm{hrs})\end{array}$ & $\begin{array}{c}t_{r}^{\operatorname{comp} p} \\
(\mathrm{hrs})\end{array}$ & Workers $\left(\left|W^{5}\right|\right)$ & $\frac{|A|}{\left|W^{5}\right|}$ & $\begin{array}{c}t_{r}^{a v g} \\
(\mathrm{hrs})\end{array}$ & $\begin{array}{c}t_{r}^{\text {comp }} \\
\text { (hrs) }\end{array}$ \\
\hline $\mathrm{P} 1$ & 293 & 6 & 49 & 18,44 & 24,29 & 6 & 49 & 18,41 & 24,12 \\
\hline $\mathrm{P} 2$ & 312 & 6 & 52 & 19,87 & 24,24 & 6 & 52 & 19,88 & 24,24 \\
\hline P3 & 295 & 5 & 59 & 22,98 & 24,42 & 6 & 49 & 19,04 & 24,33 \\
\hline $\mathrm{P} 4$ & 324 & 7 & 46 & 16,92 & 21,30 & 6 & 54 & 19,79 & 24,39 \\
\hline P5 & 333 & 7 & 48 & 18,33 & 24,24 & 7 & 48 & 18,27 & 24,26 \\
\hline P6 & 353 & 6 & 59 & 23,05 & 24,44 & 7 & 50 & 19,65 & 24,32 \\
\hline P7 & 273 & 6 & 46 & 17,53 & 23,85 & 5 & 55 & 21,14 & 24,40 \\
\hline P8 & 482 & 7 & 69 & 27,85 & 32,45 & 10 & 48 & 19,26 & 24,40 \\
\hline P9 & 311 & 7 & 44 & 17,00 & 21,64 & 6 & 52 & 19,92 & 24,34 \\
\hline P10 & 312 & 6 & 52 & 19,73 & 24,38 & 6 & 52 & 19,67 & 24,31 \\
\hline P11 & 267 & 7 & 38 & 14,50 & 16,44 & 5 & 53 & 20,22 & 24,35 \\
\hline P12 & 307 & 7 & 44 & 17,12 & 22,46 & 6 & 51 & 19,97 & 24,33 \\
\hline P13 & 345 & 6 & 58 & 21,58 & 24,43 & 7 & 49 & 18,48 & 24,21 \\
\hline P14 & 383 & 8 & 48 & 18,31 & 24,45 & 8 & 48 & 18,27 & 24,57 \\
\hline P15 & 205 & 4 & 51 & 18,65 & 24,06 & 4 & 51 & 18,74 & 24,22 \\
\hline P16 & 364 & 6 & 61 & 23,35 & 24,45 & 7 & 52 & 20,08 & 24,25 \\
\hline P17 & 269 & 5 & 54 & 20,77 & 24,30 & 5 & 54 & 20,78 & 24,27 \\
\hline P18 & 229 & 5 & 46 & 16,42 & 17,35 & 5 & 46 & 16,36 & 17,06 \\
\hline \multirow[t]{2}{*}{ P19 } & 159 & 4 & 40 & 13,85 & 16,31 & 3 & 53 & 18,49 & 23,03 \\
\hline & 5816 & 115 & & & & 115 & & & \\
\hline
\end{tabular}

Table 3. Impact of workforce transfer

to examination of their optimal support of the organization's objectives. Therefore we developed a general model of mobile process environments capable of answering questions relevant for strategic decisions by means of simulation. We could show that our model is suitable for the simulation of mobile environments and thus can predict the outcomes of regional restructuring and employee qualification efforts. We are confident, that further evolution of our model will allow for the answering of further questions, e.g. the application of automated workforce scheduling. Our long term work aims at the ability of our model not just being able to analyze scenarios, but to produce optimized scenarios based on provided cost functions.

We will keep utilizing CPN Tools for the development of our models of mobile process environments. Since we experienced difficulties to manipulate simulation data (especially with the modification of regional subdivisions) and consider our model not to be suitable for out-of-the-box use in real-world industry projects, we are currently developing a Java-based interface for the manipulation of input data and the control of simulation runs. We will further enhance the model to handle working teams of two and more resources, prioritized work items, and non-human mobile resources as e.g. technical equipment.

The assignment of the resources is currently performed by CPN ML functions in the CPN Tools suite.
Since several approaches to the assignment of mobile resources are well understood for logistics services, an interface is required to be able to utilize existing implementations of such algorithms. Especially the utilization of Java programs would decrease implementation efforts and increase the overall performance of the simulation runs. An appropriate interface is currently under development. The simplification to calculate the travel times based on constant average speeds on spherical arcs can be improved in two ways: (i) an interface to a professional route planning system could be implemented or (ii) an algorithm to automatically determine and validate the average travel speed based on the organization's real data could be developed. Due to performance issues of professional route planing systems we tend to choose the latter option.

\section{Acknowledgements}

The Chair of Applied Telematics/e-Business at the University of Leipzig is endowed by Deutsche Telekom AG.

\section{References}

[1] M. Aguilar, T. Rautert, and A. J. G. Pater. Business process simulation: a fundamental step supporting process centered management. In P. A. Farrington, H. B. Nembhard, D. T. Sturrock, and G. W. Evans, editors, 


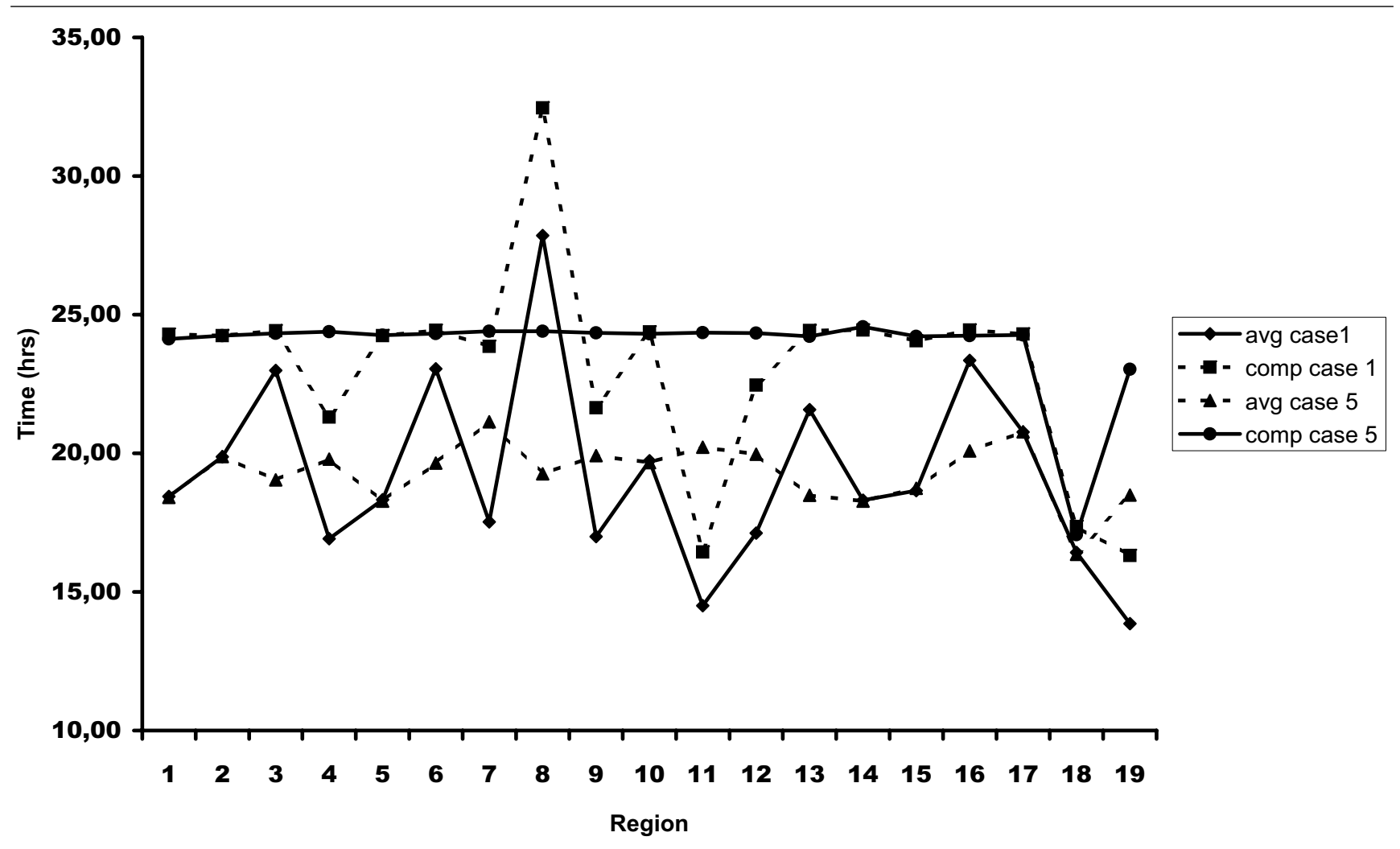

Figure 6. Impact of workforce transfer

Proceedings of the 1999 Winter Simulation Conference (WSC'99), volume 2, pages 1383-1392. IEEE Computer Society, 1999.

[2] C. Becker and F. Dürr. On location models for ubiquitous computing. Personal and Ubiquitous Computing, $9(1): 20-31,2005$

[3] G. Berio, V. Anaya, and A. Ortiz. Supporting Enterprise Integration through a Unified Enterprise Modeling Language. In Proceedings of EMOI Workshop, joint with CaiSE'04, 2004.

[4] G. Berio, K. Mertins, and F. W. Jaekel. Common Enterprise Modelling Framework for Distributed Organisations. In P. Horácek, M. Simandl, and P. Zítek, editors, Preprints of the 16th IFAC World Congress, 2005.

[5] G. A. Bolcer. Magi: an architecture for mobile and disconnected workflow. Internet Computing, IEEE, $4(3): 46-54,2000$.

[6] V. Bosilj Vuksic, J. Jaklic, and A. Popovic. Business Process Change and Simulation Modelling. Systems Integration, pages 29-, 2005.

[7] V. Bosilj Vuksic, M. I. Stemberger, J. Jarklic, and A. Kovacic. Assessment of E-Business Transformation Using Simulation Modeling. SIMULATION, 78(12):731-744, 2003.

[8] S. L. Bowden, A. Dorr, T. Thorpe, and C. J. Anumba. Mapping site processes for the introduction of mobile IT.
In A. Dikbas and R. Scherer, editors, eWork and eBusiness in architecture, engineering and construction: Proceedings of the 5th European Conference on Product and Process Modelling in the Building and Construction Industry - ECPPM 2004, 8 - 10 September 2004, Istanbul, Turkey, pages 491-498. Taylor \& Francis, 2004.

[9] T. M. Cover and P. E. Hart. Nearest Neighbor Pattern Classification. IEEE Transactions on Information Theory, 13(1):21-27, 1967.

[10] G.-J. de Vreede, A. Verbraeck, and D. T. T. van Eijck. Integrating the Conceptualization and Simulation of Business Processes: A Modeling Method and Arena Template. SIMULATION, (79):43-55, 2003.

[11] G. Doumeingts, B. Vallespir, B. Zanettin, and D. Chen. GIM, GRAI Integrated Methodology - A methodology for designing CIM systems, Version 1.0, volume Unnumbered report. 1992.

[12] S. Dustdar and H. Gall. Architectural concerns in distributed and mobile collaborative systems. Journal of Systems Architecture, 49(10-11):457-473, 2003.

[13] G. M. Giaglis, R. J. Paul, and V. Hlupic. Integrating simulation in organizational design studies. International Journal of Information Management, 19(3):219236, 1999.

[14] V. Gruhn and A. Köhler. A Modeling and Analysis Approach for Mobile Information Systems. In The First In- 
ternational Workshop on Mobility, Collaborative Working, and Emerging Applications (MobCops 2006). 2006.

[15] V. Gruhn and C. Schäfer. Determining Runtime Properties of Mobile Software Architectures. In Proceedings of the 3rd International Conference on Interoperability for Enterprise Software and Applications (I-ESA 2007), 2007.

[16] D. Innes, S. J. Barnes, and E. Scornavacca. The Impact of Wireless Field Force Automation on New Zealand Trade Services Organizations. In Proceedings of the International Conference on Mobile Business (ICMB'05), volume 00, pages 49-55. IEEE Computer Society, 2005.

[17] K. Jensen. Coloured Petri nets (2nd ed.): basic concepts, analysis methods and practical use volume 1. SpringerVerlag, London, UK, 1996.

[18] V. Kadyte. Process Visibility: How Mobile Technology Can Enhance BusinessCustomer Care in the Paper Industry. In Proceedings of the International Conference on Mobile Business (ICMB'05), volume 00, pages 159 165. IEEE Computer Society, 2005.

[19] M. Kakihara and C.-F. Sørensen. Mobility: an extended perspective. In Proceedings of the 35th Annual Hawaii International Conference on System Sciences (HICSS'02), pages 1756-1766. 2002.

[20] K. Kosanke, F. B. Vernadat, and M. Zelm. CIMOSA: enterprise engineering and integration. Computers in Industry, 40(2-3):83-97, 1999.

[21] A. Kumar, W. M. van der Aalst, and E. M. W. Verbeek. Dynamic Work Distribution in Workflow Management Systems: How to Balance Quality and Performance. Journal of Management Information Systems, 18(3):157-193, 2002.

[22] D. Lesaint, C. Voudouris, N. Azarmi, I. Alletson, and R. Laithwaite. Field Workforce Scheduling. BT Technology Journal, 21(4):23-26, 2003.

[23] P. Luff and C. Heath. Mobility in collaboration. In Proceedings of the 1998 ACM Conference on Computer supported cooperative work, pages 305-314. ACM Press, New York, USA, 1998.

[24] F. J. Martinez Borreguero and J. Chaparro Pelaez. Spanish Mobile Banking Services: An Adoption Study. In Proceedings of the International Conference on Mobile Business (ICMB'05), volume 00, pages 274-280. IEEE Computer Society, 2005.

[25] A. May, V. Mitchell, S. Bowden, and T. Thorpe. Opportunities and challenges for location aware computing in the construction industry. In MobileHCI '05: Proceedings of the 7th international conference on Human computer interaction with mobile devices $\& 5$ services, pages 255-258. ACM, 2005.

[26] N. Melao and M. Pidd. Use of business process simulation: A survey of practitioners. Journal of the Operational Research Society, 54(1):2-10, 2003.

[27] F. F.-H. Nah, K. Siau, and H. Sheng. The value of mobile applications: a utility company study. Communications of the ACM, 48(2):85-90, 2005.
[28] M. Netjes, W. M. van der Aalst, and H. A. Reijers. Analysis of resource-constrained processes with Colored Petri Nets. In Sixth Workshop and Tutorial on Practical Use of Coloured Petri Nets and the CPN Tools, 2005.

[29] M. Pesic and W. M. van der Aalst. Modelling work distribution mechanisms using Colored Petri Nets. International Journal on Software Tools for Technology Transfer (STTT), 9(3):327-352, 2007.

[30] D. Pica, C. Sørensen, and D. Allen. On Mobility and Context of Work: Exploring Mobile Police Work. In Proceedings of the 37th Annual Hawaii International Conference on System Sciences (HICSS'04). IEEE Computer Society Press, 2004.

[31] A. Popovic, M. I. Stemberger, and J. Jaklic. Applicability of Process Maps for Simulation Modeling in Business Process Change Projects. Interdisciplinary Journal of Information, Knowledge, and Management, 1, 2006.

[32] H. Ramampiaro, A. I. Wang, C.-F. Sørensen, H. N. Le, and M. Nygrd. Requirement Indicators for Mobile Work: The MOWAHS Approach. Applied Informatics, pages 1153-1160, 2003.

[33] G.-C. Roman, G. P. Picco, and A. L. Murphy. Software Engineering for Mobility: A Roadmap. In A. Finkelstein, editor, The future of Software Engineering, pages 243-258. ACM Press, New York, USA, 2000.

[34] H. Roman, E. Jaeker, and M. Wessendorf. Erfahrungen mit Workforce Management in Versorgungsnetzen. ew - das magazin für die energie wirtschaft, 106(17-18):5661, 2007.

[35] N. Russell, W. M. van der Aalst, A. H. M. ter Hofstede, and D. Edmond. Workflow Resource Patterns. In O. Pastor and J. Falcao e Cunha, editors, Proceedings of the 17th Conference on Advanced Information Systems Engineering (CAiSE'05), volume 3520 of Lecture Notes in Computer Science, pages 216-232. Springer-Verlag, 2005 .

[36] I.-I. Task Force on Architectures for Enterprise Integration. GERAM: Generalised Enterprise Reference Architecture and Methodology: Version 1.6.3. 1999.

[37] F. Vernadat. UEML: towards a unified enterprise modelling language. International Journal of Production Research, (40:17):4309-4321, 2002.

[38] T. J. Williams. The Purdue Enterprise Reference Architecture. Computers in Industry, 24(2-3):141-158, 1994.

[39] A. M. Wilson. The role of mystery shopping in the measurement of service performance. Managing Service Quality, 8(6):414-420, 1998. 